\title{
Communication \\ Relationship between plasma Neuregulin-1 and cardiac function in patients with ST-elevation myocardial infarction
}

\author{
Paul M Haller ${ }^{1,2}$, Inês F Gonçalves ${ }^{3,4}$, Eylem Acar ${ }^{3,4}$, Bernhard Jäger ${ }^{5,6}$, Patrick M Pilz ${ }^{3,4,7}$, \\ Johann Wojta ${ }^{8,9}$, Kurt Huber ${ }^{4,5,6}$, Attila Kiss ${ }^{3,4, *}$, Bruno K Podesser ${ }^{3,4}$ \\ ${ }^{1}$ Department of Cardiology, University Heart \& Vascular Center Hamburg, 20249 Hamburg, Germany \\ ${ }^{2}$ German Center for Cardiovascular Research (DZHK), Partner Site Hamburg/Kiel/Lübeck, 22041 Hamburg, Germany \\ ${ }^{3}$ Center for Biomedical Research, Medical University of Vienna, 1090 Vienna, Austria \\ ${ }^{4}$ Ludwig Boltzmann Institute for Cardiovascular Research, 1090 Vienna, Austria \\ ${ }^{5}$ Faculty of Medicine, Sigmund Freud Private University, 1020 Vienna, Austria \\ ${ }^{6} 3$ rd Department of Internal Medicine, Cardiology and Intensive Care Medicine, Klinik Ottakring, 1160 Vienna, Austria \\ ${ }^{7}$ Stanford Cardiovascular Institute, School of Medicine, Stanford University, Stanford, CA 94305, USA \\ ${ }^{8}$ Core Facilities, Medical University of Vienna, 1090 Vienna, Austria \\ ${ }^{9}$ Department of Internal Medicine III, Division of Cardiology, Medical University of Vienna, 1090 Vienna, Austria \\ *Correspondence: attila.kiss@meduniwien.ac.at (Attila Kiss) \\ Academic Editor: Daphne Merkus \\ Submitted: 7 November 2021 Revised: 12 January 2022 Accepted: 21 January 2022 Published: 14 February 2022
}

\begin{abstract}
Background: Neuregulin-1 (NRG-1) is a stress-mediated transmembrane growth factor. Reduced myocardial damage and higher NRG1 levels upon treatment with remote ischemic conditioning (RIC) has been described in rats. However, the role of NRG-1 in patients with acute myocardial infarction (MI) is unknown. Thus, we conducted a post hoc analysis of a randomized controlled trial that tested RIC in patients with MI scheduled for primary percutaneous coronary intervention (PCI). Methods: Blood was drawn from 30 patients before RIC/PCI, within 1 hour, 4 days and 1 month later. Median left ventricular ejection fraction (LVEF) in the overall study population following MI was 48.5\%. Results: NRG-1 plasma levels decreased significantly following PCI/RIC and remained decreased up to 1 month following MI $(p<0.0001)$. We observed no association of NRG-1 with other variables, including total ischemic time, LVEF or RIC. Conclusions: Thus, we identified NRG-1 may be independently affected by MI. However, further large clinical trials are warranted to clarify this hypothesis.
\end{abstract}

Keywords: Neuregulin-1; acute myocardial infarction; biomarker; left ventricular function

\section{Introduction}

Neuregulin-1 (NRG-1) is a stress-mediated paracrine transmembrane growth factor deriving, among others, from endothelial cells $[1,2]$. Emerging evidence reveals the cardiovascular functions and benefit of NRG- $1 \beta$ and downstream signaling acts on ErbB receptors [2-4]. NRG- $1 \beta$ is a stress-mediated paracrine transmembrane growth factor derived from endothelial cells [2]. It plays an essential role in embryogenic cardiac development [3], and the dysregulation of NRG-1 $\beta$ expression is also linked to various cardiovascular diseases such as myocardial infarction (MI) and heart failure (HF) [3]. In addition, a cardiovascular benefit of human recombinant NRG-1 $\beta$ (hrNRG-1 $\beta$ ) protein application in setting of myocardial ischemia/reperfusion injury [2], cardiac fibrosis, and post-MI remodeling [4] has been reported. Subsequently, it has been found that NRG-1 $\beta$ treatment modulates nitric oxide synthesis and calcium handling in cardiomyocytes (CM) [5]. In addition, Kirabo et al. [6] demonstrated that the administration of hrNRG-1 promotes cardiac fibroblast differentiation via Akt (protein kinase B) and STAT3 signal (transducer and activator of tran- scription 3) signaling activation. The anti-inflammatory effect of NRG-1 via mechanism acts on ErbB3 signaling was reported on stimulated monocytes and subsequently a marked reduction in pro-inflammatory cytokines expression [7]. Collectively, these results confirm that the application of hrNRG-1 $\beta$ has a clear cardiac benefit, however, only few studies investigated whether NRG- $1 \beta$ expression markedly changes in patients with ischemic heart disease. In addition, there is no data whether acute MI affects NRG1 expression in humans.

In line with that, we previously demonstrated that remote ischemic conditioning (RIC), a tissue protective intervention based on short, intermitted ischemia/reperfusion episodes, stimulated NRG-1 expression in the infarcted tissue area and subsequently improved cardiac function in rats with reperfused myocardial infarction (MI) [8]. This study suggests that reduction of NRG-1 levels post MI may contribute to the progression of adverse remodeling and HF. Controversially, a recent study [9] demonstrated that the recombinant form of NRG-1 application worsened left ventricular (LV) ejection fraction (LVEF) in rats with chronic MI. 
Although the administration of human-recombinant NRG-1 to patients with HF resulted in a significant improvement in cardiac output and LVEF [10], its circulating expression and functional importance as a biomarker in progression of adverse post MI remodeling in patients with ST-elevation myocardial infarction (STEMI) is yet unclear.

\section{Aim}

Thus, we aimed to study plasma NRG-1 concentrations in patients with acute STEMI and, additionally, its association with patient characteristics, cardiac function following STEMI and RIC.

\section{Methods}

We conducted a post-hoc analysis of a randomizedcontrolled trial investigating RIC in STEMI patients, that was previously reported [11]. In brief, patients with STEMI and planned primary percutaneous coronary intervention (PCI) at presentation to the emergency department were enrolled. Among others, those with symptoms $\geq 8$ hours, neurologic disorders or cardiogenic shock were excluded. All patients provided written informed consent, the study was approved by the competent ethics committee (EK 16-0090216) and was performed according to Good Clinical Practice and the Declaration of Helsinki. Blood was drawn at presentation and 1 hour, 24 hours and 1 month after PCI to derive Ethylenediaminetetraacetic acid (EDTA) anticoagulated plasma that was kept at $-80{ }^{\circ} \mathrm{C}$ until further processing. Plasma levels of NRG-1 were determined by enzymelinked immunosorbent assay (ELISA) (Abcam, Cambridge, UK). All analyses were performed according to the manufacture's protocol and in duplicate. LVEF was assessed by echocardiography approximately 4 days after STEMI. Additionally, five healthy volunteers were recruited as part of the clinical study to investigate RIC.

\section{Statistical analysis}

We fitted mixed-models specifying the patient as random factor to account for the repeated measurements design and all other included variables as fixed factors. All models included the time of measurement as a fixed effect. We report the derived estimates of the fixed effects together with 95\% confidence intervals (CI). Additionally, comparisons within one group at two time points were performed using paired Student's $t$-test for parametric variables, which were tested using Shapiro-Wilk normality test.

\section{Results}

In total, we included 30 patients in the present analysis with available data for NRG-1. The mean age was 60.7 ( \pm 13.8) and median body-mass-index 27.2 (25th, 75th percentile: $25.6,30.8)$, respectively, and 7 patients $(23.3 \%)$ were females. Overall, smoking (previous and continued) was the most prevalent risk factor $(83.3 \%)$, followed by hy-
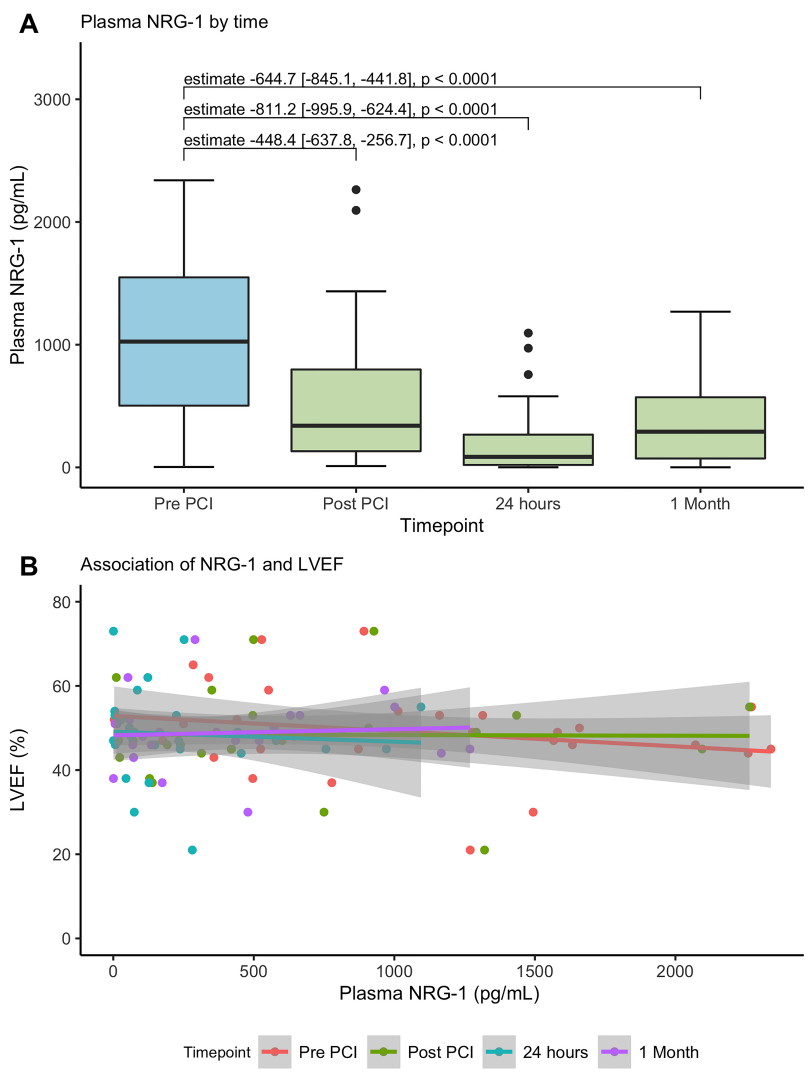

Fig. 1. Plasma Neuregulin-1 (NRG-1) levels over time (A) and in relation to left ventricular ejection fraction (LVEF; B).

pertension (53.3\%), hyperlipidemia (36.6\%) and only one patient had diabetes. Overall, two patients had known coronary artery disease. All patients did undergo primary PCI, with a median total-ischemic-time from symptom onset to vessel reopening of 185 minutes (25th, 75th percentile: 142, 304). Following STEMI, the median LVEF was $48.5 \%$ (25th, 75th percentile: $45.0,53.0$ ), with 3 patients having a LVEF $\leq 35 \%$. NRG-1 plasma levels (Fig. 1A) were highest at presentation and decreased significantly after PCI. This course was not related to the randomized group (control versus remote conditioning 142.3 [95\% CI 179.3, 470.8]), age $(-0.51[95 \% \mathrm{CI}-12.5,11.6])$, sex $(-228.2$ [95\% CI $595.6,146.6]$ ), renal function (estimated glomerular filtration rate [MDMR] 9.75 [95\% CI -10.8, 29.9]) or infarct size measures by maximum troponin release $(0.41$ [95\% CI $-0.46,1.29]$ ). Also, we did not observe a significant association with LVEF (time-adjusted estimates: pre PCI -15.4 [95\% CI -33.6, 2.8]; post PCI 12.7 [95\% CI -5.0, 30.5]; 24 hours: 11.5 [95\% CI -6.1, 29.2];1 month: 15.5 [95\% CI -6.6, 37.7]) (Fig. 1B). Additionally, NRG-1 levels were determined in five volunteers free from any known comorbidities and not taking any medication on a regular basis. In these, median NRG-1 was $758( \pm 380) \mathrm{pg} / \mathrm{mL}$. The conduction of the same RIC protocol as in STEMI patients did not significantly affect NRG-1 levels in these healthy volunteers: $823( \pm 341) \mathrm{pg} / \mathrm{mL}(p=0.6)$. 


\section{Discussion}

In summary, we investigated plasma NRG-1 concentrations for the first time in patients with acute STEMI. Although being investigated broadly in heart failure patients, NRG-1 has not been widely studied in the context of acute MI. Thus, for the first time we demonstrate that NRG-1 plasma levels are critically affected in patients with STEMI during its acute phase. Our study also highlights the importance and difference of changes in circulating NRG-1 in STEMI and HF patients. Accordingly, we found that circulating NRG-1 levels were declined after PCI in STEMI patients. On the other hand, patients with advanced stage of HF were characterized with a significant increase of circulating NRG-1 levels [12] but mRNA expression of ErbB2 and ErB4 were decreased [13].

In our previous study, we reported on the effect of RIC on NRG-1 plasma and tissue concentrations, respectively, in experimental myocardial infarction in rats [8]. Although effective in rats, we did not observe any effect of RIC on plasma NRG-1 levels. Lately, the effect or RIC in humans has been questioned [14]. Although some studies did reveal some positive effect on myocardial salvage in the context of STEMI, the overall effect seems to be modest [15]. Also, a large randomized controlled trial did not show any effect on survival or hospitalization for heart failure following in the first year after STEMI [16]. Despite the previously described association of NRG-1 and cardiac function, we did not observe any association with LVEF. Although this will at some point be owed to the small number of patients included in this analysis, the early time of LVEF determination following STEMI of only four days will most likely not fully reflect the effect of STEMI on the developing heart failure. In line, the overall reduction in LVEF observed in our trial was small, with only three patients having a LVEF $\leq 35 \%$. We did not observe an association of circulating NRG-1 levels and LVEF, however, this may be explained by the setting of the trial. Since increased levels of NRG-1 are known for HF patients, all patients in the present trial did have MI acute MI and remodeling did not have taken place completely. Also, the number of patients with severely reduced LVEF was small. Furthermore, the decrease of NRG1 may be a marker and contributor of the development of microvascular obstructions and diastolic dysfunction after MI. In line, a recent study [17] demonstrated that a catheterbased intramyocardial injection of NRG1-loaded microparticles markedly improves left ventricular diastolic function in a preclinical pig model of myocardial ischemia and reperfusion. Nevertheless, further preclinical and clinical studies are warranted to clarify this hypothesis.

In summary, our findings highly suggest a further role of NRG-1 in humans with acute MI, despite its known role in heart failure. Considering the beneficial effects of the administration of recombinant NRG-1 to patients with heart failure [10], patients with STEMI could be a further target population of interest. Future studies will need to address the function of NRG-1 during acute MI and its relation to LVEF and other cardiac parameters, in mid- and long-term follow-up.

Table 1. Provides baseline characteristics.

\begin{tabular}{lc}
\hline & Study population \\
\hline Age & $60.7(3.8)$ \\
Females & $7(23.3 \%)$ \\
Hypertension & $16(53.3 \%)$ \\
Hyperlipoproteinaemia & $11(36.6 \%)$ \\
Diabetes & $1(3.3 \%)$ \\
Smoking history & \\
Previously & $11(36.7 \%)$ \\
Continued & $14(46.7 \%)$ \\
History of Coronary Artery Disease & $2(6.7 \%)$ \\
Total ischemic time, minutes & $234.7(127.4)$ \\
TIMI flow pre PCI & $0(0,3)$ \\
TIMI flow post PCI & $3(3,3)$ \\
Maximum Creatine Kinase & $1865(122,20023)$ \\
Maximum Troponin & $72.5(0.5,975.0)$ \\
Maximum NT-proBNP & $1460.0(654.5,3515.2)$ \\
eGFR at presentation & $64.9(8.4)$ \\
BMI & $27.2(25.6,30.8)$ \\
Left ventricular ejection fraction & $48.5(45.0,53.0)$ \\
following STEMI & \\
Left ventricular ejection fraction & $2(6.7 \%)$ \\
Severe $(\leq 30 \%)$ & $7(23.3 \%)$ \\
Moderate $(31 \leq 45 \%)$ & $9(30.0 \%)$ \\
Slight $(46 \leq 50 \%)$ & $12(40.0 \%)$ \\
Normal $(>50 \%)$ & \\
\hline Categorial variables are presented as $n(\%)$ and scale vari- \\
ables as mean $( \pm$ standard deviation) or median $(25$ th and \\
75 th percentile). & \\
&
\end{tabular}

\section{Author contributions}

Conceptualization and methodology: $\mathrm{PMH}, \mathrm{AK}$, BKP, JW and KH. Formal analysis: PMH and AK. Investigation: PMH, IFG, EA, BJ, PMP, JW and KH. WritingOriginal draft preparation: $\mathrm{PMH}$ and AK. WritingReview and editing: all authors. Supervision: $\mathrm{KH}$ and BKP. Project administration: PMH, AK and KH. Funding acquisition: $\mathrm{PMH}, \mathrm{JW}, \mathrm{AK}, \mathrm{BKP}$ and $\mathrm{KH}$.

\section{Ethics approval and consent to participate}

All patients provided written informed consent, the study was approved by the competent ethics committee (EK 16-009-0216) and was performed according to Good Clinical Practice and the Declaration of Helsinki.

\section{Acknowledgment}

We would like to thank the staff of Wojta's lab for continuous advice throughout the study and the peer reviewers for their opinions and suggestions. 


\section{Funding}

This work was supported by the Ludwig Boltzmann Institute for Cardiovascular Research, by a grant of the Austria-Hungary Action Foundation (No. 920̈u8) and the Association for Research on Arteriosclerosis, Thrombosis and Vascular Biology (ATVB).

\section{Conflict of interest}

The authors declare no conflict of interest. Attila Kiss is serving as one of the Guest editors of this journal. We declare that Attila Kiss had no involvement in the peer review of this article and has no access to information regarding its peer review. Full responsibility for the editorial process for this article was delegated to Daphne Merkus.

\section{Availability of data and material}

The datasets used and/or analyzed during the current study are available from the first author on reasonable request.

\section{Appendix}

See Table 1.

\section{References}

[1] Rupert CE, Coulombe KLK. The Roles of Neuregulin-1 in Cardiac Development, Homeostasis, and Disease. Biomarker Insights. 2015; 10: 1-9.

[2] Hedhli N, Huang Q, Kalinowski A, Palmeri M, Hu X, Russell $\mathrm{RR}$, et al. Endothelium-derived neuregulin protects the heart against ischemic injury. Circulation. 2011; 123: 2254-2262.

[3] Geissler A, Ryzhov S, Sawyer D. Neuregulins: protective and reparative growth factors in multiple forms of cardiovascular disease. Clinical Science. 2020; 134: 2623-2643.

[4] Gupte M, Lal H, Ahmad F, Sawyer DB, Hill MF. Chronic Neuregulin-1beta Treatment Mitigates the Progression of Postmyocardial Infarction Heart Failure in the Setting of Type 1 Diabetes Mellitus by Suppressing Myocardial Apoptosis, Fibrosis, and Key Oxidant-Producing Enzymes. Journal of Cardiac Failure. 2017; 23: 887-899.

[5] Brero A, Ramella R, Fitou A, Dati C, Alloatti G, Gallo MP, et al. Neuregulin-1beta1 rapidly modulates nitric oxide synthesis and calcium handling in rat cardiomyocytes. Cardiovascular Research. 2010; 88: 443-452.

[6] Kirabo A, Ryzhov S, Gupte M, Sengsayadeth S, Gumina RJ, Sawyer DB, et al. Neuregulin-1beta induces proliferation, sur- vival and paracrine signaling in normal human cardiac ventricular fibroblasts. Journal of Molecular and Cellular Cardiology. 2017; 105: 59-69.

[7] Ryzhov S, Matafonov A, Galindo CL, Zhang Q, Tran T, Lenihan DJ, et al. ERBB signaling attenuates proinflammatory activation of nonclassical monocytes. American Journal of PhysiologyHeart and Circulatory Physiology. 2017; 312: H907-H918.

[8] Pilz PM, Hamza O, Gidlöf O, Gonçalves IF, Tretter EV, Trojanek $\mathrm{S}$, et al. Remote ischemic perconditioning attenuates adverse cardiac remodeling and preserves left ventricular function in a rat model of reperfused myocardial infarction. International Journal of Cardiology. 2019; 285: 72-79.

[9] Zurek M, Johansson E, Palmer M, Albery T, Johansson K, Rydén-Markinhutha K, et al. Neuregulin-1 Induces Cardiac Hypertrophy and Impairs Cardiac Performance in Post-Myocardial Infarction Rats. Circulation. 2020; 142: 1308-1311.

[10] Jabbour A, Hayward CS, Keogh AM, Kotlyar E, McCrohon $\mathrm{JA}$, England JF, et al. Parenteral administration of recombinant human neuregulin-1 to patients with stable chronic heart failure produces favourable acute and chronic haemodynamic responses. European Journal of Heart Failure. 2011; 13: 83-92.

[11] Haller PM, Jäger B, Piackova E, Sztulman L, Wegberger C, Wojta $\mathrm{J}$, et al. Changes in Circulating Extracellular Vesicles in Patients with ST-Elevation Myocardial Infarction and Potential Effects of Remote Ischemic Conditioning - A Randomized Controlled Trial. Biomedicines. 2020; 8: 218.

[12] Ky B, Kimmel SE, Safa RN, Putt ME, Sweitzer NK, Fang JC, et al. Neuregulin-1 beta is associated with disease severity and adverse outcomes in chronic heart failure. Circulation. 2009; 120: 317-317.

[13] Rohrbach S, Niemann B, Silber RE, Holtz J. Neuregulin receptors erbB2 and erbB4 in failing human myocardium. Basic Research in Cardiology. 2005; 100: 240-249.

[14] Hausenloy DJ, Botker HE. Why did remote ischaemic conditioning not improve clinical outcomes in acute myocardial infarction in the CONDI-2/ERIC-PPCI trial? Cardiovasc Research. 2019; 115: e161-e163.

[15] Haller PM, Vargas KG, Haller MC, Piackova E, Wojta J, Gyöngyösi $\mathrm{M}$, et al. Remote ischaemic conditioning for myocardial infarction or elective PCI: systematic review and metaanalyses of randomised trials. European Heart Journal: Acute Cardiovascular Care. 2020; 9: 82-92.

[16] Hausenloy DJ, Kharbanda RK, Møller UK, Ramlall M, Aarøe $\mathrm{J}$, Butler R, et al. Effect of remote ischaemic conditioning on clinical outcomes in patients with acute myocardial infarction (CONDI-2/ERIC-PPCI): a single-blind randomised controlled trial. The Lancet. 2019; 394: 1415-1424.

[17] Garbayo E, Gavira JJ, de Yebenes MG, Pelacho B, Abizanda G, Lana $\mathrm{H}$, et al. Catheter-based Intramyocardial Injection of FGF1 or NRG1-loaded MPs Improves Cardiac Function in a Preclinical Model of Ischemia-Reperfusion. Scientific Reports. 2016; 6: 25932 . 